Bundesgesundheitsbl 2010 · 53:510-519 DOI 10.1007/s00103-010-1074-3

Online publiziert: 2. Mai 2010

(c) Springer-Verlag 2010

G. Krause ${ }^{1} \cdot$ A. Gilsdorf ${ }^{1} \cdot$ J. Becker ${ }^{2} \cdot$ K. Bradt ${ }^{3} \cdot$ C. Dreweck ${ }^{4}$ B. Gärtner ${ }^{5}$.

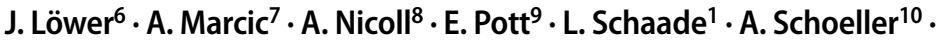
V. Stollorz ${ }^{11} \cdot$ C. Träder $^{12} \cdot 0$. Razum $^{13}$

${ }^{1}$ Abteilung für Infektionsepidemiologie, Robert Koch-Institut, Berlin

${ }^{2}$ Ruhland, Berlin

${ }^{3}$ Landesinstitut für Gesundheit und Arbeit NRW, Düsseldorf

${ }^{4}$ Referat für Gesundheit und Umwelt, Landeshauptstadt München, München

${ }^{5}$ Universitätsklinikum des Saarlandes, Homburg/Saar

${ }^{6}$ Bundesinstitut für Arzneimittel und Medizinprodukte, Bonn

${ }^{7}$ Ministerium für Arbeit, Soziales und Gesundheit, Schleswig-Holstein, Kiel

${ }^{8}$ European Centre for Disease Prevention and Control (ECDC), Stockholm

${ }^{9}$ Bundeszentrale für gesundheitliche Aufklärung, Köln

${ }^{10}$ Bundesärztekammer, Berlin

${ }^{11}$ Wissenschaftsjournalist, Köln

${ }^{12}$ Vivantes Auguste-Viktoria-Klinikum, Berlin

${ }^{13}$ Fakultät für Gesundheitswissenschaften, Universität Bielefeld, Bielefeld

\title{
Erster Erfahrungsaustausch
} zur H1N1-Pandemie in Deutschland 2009/2010

\section{Bericht über einen Workshop am 22. und 23. März 2010 in Berlin}

Ländern mit zunächst nur einer geringen Zahl autochthoner Infektionen. Ab dieser Zeit wurden die Infektionsschutzmaßnamen auf vulnerable Gruppen begrenzt [4]. Nachdem im August die wöchentlichen registrierten Meldungen zunächst wieder sanken, stiegen die Fallzahlen ab Oktober zuerst in Süddeutschland erneut an, und erreichten Anfang November deutschlandweit einen Höhepunkt mit bis zu 45.000 neu gemeldeten Fällen pro Woche.

Auch die im Sentinel der Arbeitsgemeinschaft Influenza gemessene Influenzaaktivität überschritt im Oktober erstmals den Bereich der Hintergrundaktivität und bestätigte das Bild einer pandemischen Welle mit autochthoner Übertragung und messbarer Krankheitslast auf Bevölkerungsebene. Mit Beginn dieser Welle wurden die Infektionsschutz- und Surveillance-Maßnahmen im Wesentlichen auf die Empfehlungen reduziert, die auch bei saisonaler Influenza Gültigkeit haben. Im Oktober 2009 begann die
Impfkampagne gegen das pandemische Influenzavirus ( $\left.\mathrm{H}_{1} \mathrm{~N}_{1}\right) 2009$ in Deutschland. Ab der 48. Kalenderwoche ging die Aktivität von akuten Atemwegserkrankungen bundesweit stark zurück. Insgesamt wurden dem Robert Koch-Institut (RKI) bis März 2010 über 220.00o Fälle von pandemischer Influenza $\left(\mathrm{H}_{1} \mathrm{~N}_{1}\right)$ 2009 übermittelt. Die wahre Zahl der Infizierten dürfte jedoch um ein Vielfaches höher liegen. In Deutschland wurden über 250 Todesfälle im Zusammenhang mit einer nachgewiesenen Infektion mit pandemischer Influenza ( $\left.\mathrm{H}_{1} \mathrm{~N}_{1}\right) 2009$ erfasst [5]. Im Gegensatz zur saisonalen Influenza war die Mortalität bei Säuglingen und den Erwachsenen zwischen 35 und 59 Jahren mit rund vier Todesfällen pro einer Millionen Einwohner nahezu doppelt so hoch wie in anderen Altersgruppen [5]. Vorläufige Schätzungen des RKI gehen davon aus, dass Infektionen mit pandemischer Influenza (H1N1) 2009 in der Herbstwelle 2009 1,8 bis 3,5 Millionen zusätzliche Arztbesuche in Deutsch- 
land verursachten (95\% Konfidenzintervall), was im mittleren Bereich saisonaler Influenzawellen der vergangenen drei Jahre liegt (o,3 bis 5,o Millionen).

Das pandemische Influenzavirus (H1N1) 2009 löste die erste Pandemie im 21. Jahrhundert aus. Aufgrund der Dauer des Infektionsgeschehens und der Intensität der getroffenen Maßnahmen kann der Zeitraum vom Bekanntwerden des neuen Influenzavirus bis zur ersten pandemischen Welle und der zeitgleich begonnenen Impfkampagne als eine der größten Herausforderungen der vergangenen Jahrzehnte für den Öffentlichen Gesundheitsdienst (ÖGD) bewertet werden. Auch wenn und gerade weil die Pandemie mit dem pandemischen Influenzavirus $\left(\mathrm{H}_{1} \mathrm{~N}_{1}\right)$ 2009 global noch nicht beendet ist, erscheint es notwendig, zeitnah einen Prozess der Aufarbeitung und Anpassung der Pandemieplanung einzuleiten und Konsequenzen für die Zukunft zu ziehen. Hierzu organisierte das RKI einen Erfahrungsaustausch mit dem Ziel, einen Beitrag für weiterführende Auswertungen zu leisten, die auf örtlicher, Landes- und Bundesebene sowie in nationalen Verbänden und internationalen Gremien und Organisationen erfolgen werden.

\section{Methoden}

Das RKI hatte Vertreterinnen und Vertreter der Ärzteschaft, verschiedener Berufsund Fachverbände sowie Behörden, Ministerien und Journalisten eingeladen, die an der Planung und Umsetzung von Maßnahmen im Rahmen einer Pandemie in besonderer Weise beteiligt sind (Teilnehmerliste siehe unten). Aus den im Vorfeld von den Teilnehmern eingereichten Themenvorschlägen ergaben sich vier Themengebiete „Maßnahmen“, „Koordination“, „Impfung“ und „Kommunikation“. Die 85 Teilnehmer wurden entsprechend ihrer Expertise und der von ihnen eingereichten Themenvorschläge einer dieser Gruppen zugeteilt. Die Diskussionen wurden von einem Mitglied der Arbeitsgruppe moderiert und einem Mitarbeiter des RKI protokolliert.

Als Anregung für die Diskussion in den Arbeitsgruppen und im Plenum dienten neun Kurzvorträge, ergänzt durch den Vortrag eines Vertreters des Euro-

Bundesgesundheitsbl 2010 ·53:510-519 DOI 10.1007/s00103-010-1074-3

(c) Springer-Verlag 2010

\section{G. Krause · A. Gilsdorf · J. Becker · K. Bradt · C. Dreweck · B. Gärtner · J. Löwer · A. Marcic · A. Nicoll · E. Pott · L. Schaade · A. Schoeller · V. Stollorz · C. Träder · O. Razum Erster Erfahrungsaustausch zur H1N1-Pandemie in Deutschland 2009/2010. Bericht über einen Workshop am 22. und 23. März 2010 in Berlin}

\section{Zusammenfassung}

Im April 2009 entwickelte sich innerhalb we-

niger Wochen von Mexiko ausgehend die erste Influenzapandemie des 21. Jahrhunderts, deren erste Welle Deutschland im Herbst 2009 erreichte und 1,8 bis 3,5 Millionen zusätzliche Arztbesuche verursachte. Für den Öffentlichen Gesundheitsdienst war diese Pandemie eine der größten Herausforderungen der vergangenen Jahrzehnte. Als erster Beitrag für weiterführende Auswertungen auf nationaler und internationaler Ebene hat das Robert Koch-Institut Vertreter unterschiedlicher Berufsgruppen und Institutionen vom 22. bis 23. März 2010 zu einem Erfahrungsaustausch mit Kurzvorträgen, Gruppenund Plenardiskussionen eingeladen. Wesentliche Erfahrungen sind, dass a) die Pandemiepläne hilfreich, b) die Frühwarnsysteme zuverlässig und c) Impfstoffe innerhalb weni- ger Monate begrenzt verfügbar waren. Verbesserungsbedarf wurde vor allem diskutiert bezüglich a) der Effizienz der Impfstofflogistik, b) der Regelung der Impfkostenerstattung, c) der Verfügbarkeit von Surveillanceund Monitoring-Systemen, d) der Einbindung der Ärzteschaft in Entscheidungsprozesse und Aufklärungsarbeit sowie e) einer vorausschauenden Öffentlichkeitsarbeit. Entsprechende Investitionen können den gesundheitlichen Schutz der Bevölkerung für die Zukunft verbessern.

Schlüsselwörter Pandemische Influenza (H1N1) 2009.

Schweinegrippe - Pandemie - Evaluation . Öffentlicher Gesundheitsdienst

\section{First exchange of experiences concerning the H1N1 pandemic in Germany 2009/2010. Report on a workshop held March 22-23, 2010, in Berlin}

\section{Abstract}

In April 2009 the first pandemic of the 21st century developed within a few weeks starting from Mexico. Its first wave reached Germany in autumn 2009 and was responsible for 1.8-3.5 million additional medical consultations. For the public health sector, this pandemic was one of the largest challenges of the last few decades. As a contribution to broader evaluations on national and international level the Robert Koch Institute invited representatives from different professions involved in the pandemic response to participate in a workshop on 22-23 March 2010. This workshop was structured in short presentations, group work, and plenary discussions. The Main experiences were that (a) pandemic preparedness was helpful, (b) the early warning systems were reliable, (c) vac- cines were available within a few months, however, in limited amounts. Need for improvement was discussed for (a) effectiveness of vaccination logistics, (b) mechanisms for the reimbursement of the cost of vaccination, (c) availability of surveillance and monitoring systems, (d) integration of physicians in decision-making processes and health education, and (e) proactive communication strategies. Investments in the above mentioned areas can help to improve public health protection in the future.

\section{Keywords}

Pandemic (H1N1) 2009 . Swine flu .

Pandemic · Evaluation · Public health service 


\section{Bundesministerien}

Dr. Enno Winkler, Auswärtiges Amt, Berlin; Franz-Josef Bindert, Bundesministerium für Gesundheit, Berlin (BMG); Dr. Johannes Blasius, BMG; Ministerialdirektorin Karin Knufmann-Happe, BMG; Sophia Matenaar, BMG; Prof. Dr. Michael Kramer, BMG; Andreas Lemmer, BMG; Arnold Schreiber, BMG; Lutz Sindermann, BMG; René Du Bois, Bundesministerium des Inneren (BMI),Berlin; Harald Vorpahl, BMI; Dr. Andrea Joram-Savoy, Bundesministerium der Verteidigung, Bonn

\section{Weitere Bundeseinrichtungen}

Regine Lehnert ${ }^{\mathrm{a}}$, Bundesinstitut für Arzneimittel und Medizinprodukte, Bonn (BfArM); Prof. Dr. Johannes Löwer ${ }^{\mathrm{d}}$, BfArM; Prof. Dr. Elisabeth Pott ${ }^{\mathrm{d}}$, Bundeszentrale für gesundheitliche Aufklärung, Köln (BZgA); Dr. Monika von dem Knesebeck, BZgA; Dr. Timm Hardera , Friedrich-Loeffler-Institut, Greifswald - Insel Riems; Prof. Dr. Klaus Cichutek, Paul-Ehrlich-Institut, Langen (PEl); Dr. Brigitte Keller-Stanislawski, PEl; Dr. Susanne Stöcker, PEl; Dr. Ralf Wagner, PEl; Dr. Walter Biederbick, Robert Koch-Institut, Berlin (RKI); Dr. Udo Buchholz, RKI; Dr. Silke Buda, RKI; Prof. Dr. Reinhard Burger, RKI; Günther Dettweiler, RKI; Dr. Tim Eckmanns, RKI; Susanne Glasmacher, RKI; PD Dr. Walter Haas, RKI; Dr. Osamah Hamouda, RKI; PD Dr. Gérard Krause ${ }^{\text {d }}$, RKI; Claudia Lerch, RKI; Dr. Dorothea Matysiak-Klose, RKI; Prof. Dr. Martin Mielke, RKI; Dr. Alfred Nassauer, RKI; Dr. Sabine Reiter, RKI; Annicka Reuß, RKI; PD Dr. Lars Schaaded, RKI; Dr. Brunhilde Schweiger, RKI; PD Dr. Thorsten Wolff, RKI

\section{Landes- und Kommunalbehörden}

Heidrun Böhm, Sächsisches Staatsministerium für Soziales, Dresden; Kirsten Bradte, Landesinstitut für Gesundheit und Arbeit NRW, Düsseldorf; Enno Gosling, Ministerium für Soziales Frauen, Familie und Gesundheit, Hannover; Dr. Klaus Jahn, Hessisches Ministerium für Arbeit, Familie und Gesundheit, Wiesbaden; Dr. Regine Kämmerer, Ministerium für Arbeit, Gesundheit und Soziales des Landes NRW, Düsseldorf; Dr. Ingeborg Kirchhoff, Behörde für Soziales, Familie, Gesundheit und Verbraucherschutz, Amt für Gesundheit und Verbraucherschutz, Hamburg; Dr. Renate Klein, Ministerium für Justiz, Arbeit, Gesundheit und Soziales des Saarlandes, Saarbrücken; Prof. Dr. Bijan Kouros, Ministerium für Arbeit und Soziales Baden-Württemberg, Stuttgart; Prof. Dr. Bernhard R. Liebl, Bayerisches Staatsministerium für Umwelt, Gesundheit und Verbraucherschutz, München; Dr. Anne Marcic ${ }^{\mathrm{d}}$, Ministerium für Arbeit, Soziales und Gesundheit des Landes Schleswig-Holstein, Kiel; Dr. Sonja Nowak, Landesgesundheitsamt Brandenburg, Zossen; Dr. Isolde Piechotowski, Landesgesundheitsamt Baden-Württemberg, Stuttgart; Dr. Matthias Pulz, Niedersächsisches Landesgesundheitsamt, Hannover; Stefanie Schumacher, Ministerium für Soziales und Gesundheit des Landes Mecklenburg-Vorpommern, Schwerin; Dr. Eike Schumann, Ministerium für Arbeit, Soziales, Gesundheit, Familie und Frauen des Landes Rheinland-Pfalz, Mainz; Dr. Marlen Suckau, Senatsverwaltung für Gesundheit, Umwelt und Verbraucherschutz, Berlin; Dr. Ulrich Widders, Ministerium für Umwelt, Gesundheit und Verbraucherschutz, Potsdam; Dr. Heidemarie Willer, Ministerium für Gesundheit und Soziales des Landes Sachsen-Anhalt, Magdeburg; Dr. Maren Wölk, Thüringer Ministerium für Soziales, Familie und Gesundheit, Erfurt; Dr. Caroline Dreweck ${ }^{\mathrm{d}}$, Referat für Gesundheit und Umwelt, Landeshauptstadt München; PD Dr. René Gottschalka, Amt für Gesundheit, Frankfurt/Main; Dr. Ronald Woltering, Gesundheitsamt Höxter, Höxter

\section{Klinisch tätige Ärzteschaft}

Prof. Dr. Heinz-Harald Abholz, Universitätsklinikum Düsseldorf, Deutsche Gesellschaft für Allgemeinmedizin und Familienmedizin; Dr. Johannes Beckerd, Niedergelassener Arzt, Ruhland; Prof. Dr. Barbara Gärtnera, ,Universitätsklinikum des Saarlandes, Homburg/Saar; Dr. Herbert Grundhewer, Niedergelassener Arzt, Berlin; Prof. Dr. Friedrich Hofmann ${ }^{b}$, Bergische Universität Wuppertal; Dr. Lothar Maurer, Niedergelassener Arzt, Frankenthal; Prof. Dr. Thomas Mertens ${ }^{\mathrm{a}, \mathrm{b}}$, Universität Ulm; Prof. Dr. Georg Peters ${ }^{\mathrm{a}, \mathrm{c}}$, Universität Münster; Dr. Andreas Petri, Niedergelassener Arzt, Hürth; Prof. Dr. Bernhard R. Rufa, Klinikum St. Georg, Leipzig; Dr. Christian Träder ${ }^{d}$, Vivantes Auguste-Viktoria-Klinikum, Berlin; Prof. Dr. Angelika Vallbracht ${ }^{c}$, Universität Bremen; Dr. Steffen Weber-Carstens, Charité, Berlin

\section{Andere Berufsgruppen, Verbände und Institutionen}

Prof. Dr. Jörg Hacker, Deutsche Akademie der Naturforscher Leopoldina - Nationale Akademie der Wissenschaften, Halle, bis Februar 2010 Präsident des RKI; Dr. Iris Juditzki, Deutsche Krankenhausgesellschaft e.V., Berlin; Dr. Jörn Knöpnadel, Kassenärztliche Bundesvereinigung, Berlin; Dr. Thomas Menn, Bundesverband der Ärztinnen und Ärzte des öffentlichen Gesundheitsdienstes, Potsdam; Dr. Annette Nahnhauer, GKV-Spitzenverband, Berlin; Prof. Angus Nicoll $^{d}$, European Centre for Disease Prevention and Control (ECDC), Stockholm; Prof. Dr. Oliver Razume ${ }^{\mathrm{e}}$, Universität Bielefeld, Vorsitzender der Deutschen Gesellschaft für Epidemiologie; Dr. Annegret Schoeller ${ }^{\mathrm{a}, \mathrm{e}}$, Bundesärztekammer, Berlin; Volker Stollorz ${ }^{\mathrm{d}, \mathrm{e}}$, freier Wissenschaftsjournalist, Köln; Dr. Vera ZylkaMenhorn, Deutsches Ärzteblatt, Köln

aMitglied der Influenzakommission für den Pandemiefall

${ }^{b}$ Mitglied der Ständigen Impfkommission

'Mitglied des Wissenschaftlichen Beirates des RKI

${ }^{d}$ Referent/in eines Vortrages im Workshop

eModerator/in einer Arbeitsgruppe beziehungsweise im Plenum

pean Centre for Disease Prevention and Control (ECDC) in Stockholm, der Erfahrungen und Daten aus anderen Ländern der Europäischen Union (EU) vorstellte. Der vorliegende Bericht ist in die drei Kapitel „Infektionsschutzmaßnahmen und Koordination“, „Impfung“ und „Kommunikation" gegliedert, in denen die je- weiligen Aspekte aus Kurzvorträgen, Arbeitsgruppenberichten und Plenardiskussion zusammengeführt werden.

Autoren dieses Berichtes sind die Referenten der Kurzvorträge, die Moderatoren der Arbeitsgruppen sowie der Moderator der Plenardiskussionen und ein für die Gestaltung des Workshops zustän- diger RKI-Wissenschaftler. Die im Folgenden dargestellten Ergebnisse repräsentieren daher nicht notwendigerweise die Meinung aller beteiligten Institutionen und Teilnehmer, spiegeln aber nach Auffassung der Autoren die im Workshop geführte Diskussion wider. 


\section{Ergebnisse}

\section{Infektionsschutzmaßnahmen und Koordination}

\section{Anwendung des Pandemieplans}

Es erwies sich als nützlich, auf die Pandemieplanung zurückgreifen zu können, wenngleich in Planungsszenarien sowie in der nationalen Pandemie-Übung LÜKEX im Jahr 2007 von einem schwerwiegenderen Verlauf ausgegangen wurde $[6,7]$. Das Infektionsschutzmanagement wurde insbesondere in den ersten Wochen als positiv und wirksam empfunden. In kürzester Zeit waren ÖGD und Ärzteschaft informiert und fachliche Empfehlungen und Informationen abgestimmt. Die vielfältigen Informationsmaterialien wurden als sehr hilfreich empfunden. Das anfänglich bewusst strenge Infektionsschutzkonzept erforderte erhebliche Ressourcen. So musste beispielsweise das Gesundheitsamt München in den ersten Wochen des Einzelfallmanagements für einen bestätigten Fall zehn bis 50 Arbeitsstunden für die Ermittlungen und die ausführliche Information der Erkrankten und ihres Umfelds aufwenden. Spätestens in der Herbstwelle waren die personellen Ressourcen des ÖGD bis an die Grenze der Belastbarkeit beansprucht.

Entsprechend der im Pandemieplan enthaltenden Flexibilität wurde versucht, die Maßnahmen bedarfsorientiert und an die Situation angepasst umzusetzen. Einige im Pandemieplan erwähnten Maßnahmen wurden in Deutschland bewusst nicht getroffen: So wurden Schulen lediglich als Reaktion auf Ausbrüche, nicht aber präventiv geschlossen. Im Vergleich zu den Regelungen anderer Länder der EU wurde zum Beispiel die antivirale Medikation gezielter eingesetzt. Allerdings hat die Ärzteschaft insbesondere die Arbeitsschutzempfehlungen teilweise als überzogen empfunden.

\section{Deeskalation}

Die Entscheidung zur Deeskalation, das heißt zur Reduzierung und Vereinfachung von Maßnahmen, fiel hingegen aus Sicht vieler Beteiligter zu spät. So wurde zum Beispiel die bereits am 2. Mai 2009 eingeführte Meldepflicht für Verdachtsfälle erst am 14. November 2009 aufgehoben
[8]. Eine zur Erleichterung der Gesundheitsämter in der 46. Kalenderwoche eingeführte Möglichkeit zur aggregierten Fall-Übermittlung hat sich nur bedingt bewährt.

Eine frühzeitige Einigung über die Deeskalation wurde zum Teil auch aufgrund regional unterschiedlicher Lagen verzögert und auch von der Sorge bestimmt, eine Reduktion des Infektionsschutzes, der Surveillance oder der Berichtsfrequenz könnten implizit entweder als verfrühte „Entwarnung“ oder als ein „Zusammenbrechen der Kapazitäten“ missverstanden werden.

ÖGD und Ärzteschaft hätten für einen Wechsel der Maßnahmen mehr Vorlaufzeit benötigt, und die Strategien hätten über die im Epidemiologischen Bulletin beschriebene Strategieanpassung hinaus durch kurze Botschaften an die Öffentlichkeit besser verständlich gemacht werden müssen [9]. Unabhängig von der Diskussion um die Deeskalation bestand Einigkeit, dass auch nach dem ersten Erkrankungsgipfel Wachsamkeit geboten ist.

\section{Informationsbedarf}

Insbesondere zu Beginn der Pandemie bestand ein extrem hoher Informationsbedarf, der nur teilweise durch die bestehenden Surveillanceinstrumente (zum Beispiel das Meldewesen nach Infektionsschutzgesetz, IfSG) gedeckt werden konnte. Es fehlte an Informationsstrukturen für die Erfassung schwerer Krankheitsverläufe und der Gesamtmortalität. Diese Informationslücke hat möglicherweise in der allgemeinen Wahrnehmung dazu geführt, die klinische Bedeutung einer Infektion mit der pandemischen Influenza $\left(\mathrm{H}_{1} \mathrm{~N}_{1}\right) 2009 \mathrm{zu}$ unterschätzen.

Es bestand Einigkeit darüber, dass neue Surveillanceinstrumente nicht effektiv während eines Ereignisses etabliert werden können. Die Versuche, die erforderlichen Informationen über aufwendige Zusatzerhebungen und improvisierte Freitextübermittlungen im Rahmen der derzeitigen Übermittlungs- und IT-Strukturen des Infektionsschutzgesetzes zu erfassen, erwiesen sich als wenig geeignet und zu personalintensiv. Um das Meldepflichtsystem entlasten zu können, müssen Übermittlungsprozesse beschleunigt und weiter automatisiert werden. Auch das kurzfristig aufgebaute pandemische Influenza-Krankenhaus-Surveillancesystem (PIKS) kam zu spät und war nicht repräsentativ. Intensivierte Studien, wie die im Sommer durchgeführte RKI-Studie zur Virusübertragbarkeit, können die Erfassungslast in der Fläche reduzieren. Ebenso notwendig erscheint eine zeitnahe Mortalitätssurveillance, die vermutlich eine Änderung gesetzlicher Regelungen erfordert. Es wurde aber auch deutlich, dass in der akuten Lage trotz mehrjähriger Abstimmungen eines Instrumentes für Pandemie-Lageindikatoren im Herbst 2009 kein flächendeckendes oder zumindest repräsentatives Monitoring über die Auslastung der medizinischen Versorgungsstrukturen und des ÖGD anwendbar war.

Die Diskussion machte ferner deutlich, dass die Ärzteschaft und bisweilen auch der ÖGD bisher nur unzureichend erkennen, dass sich die Meldepflicht-Surveillance nicht nur durch Infektionsschutzmaßnahmen bezüglich des betreffenden Individuums rechtfertigt. Vielmehr dient sie auch dazu, Infektionsschutzmaßnahmen bis hin zu Impfempfehlungen auf der Bevölkerungsebene zu definieren und gegebenenfalls anzupassen. Die jeweils unterschiedlichen Anliegen der auf das Individuum bezogenen klinischen Medizin einerseits und den bevölkerungsbezogenen Aufgaben des ÖGD andererseits müssen dabei besser berücksichtigt werden.

\section{Medizinische Versorgung}

Der hohe medizinische Standard und die guten labordiagnostischen Kapazitäten in Deutschland haben vermutlich zur Vermeidung von Todesfällen beigetragen. Aber es ist bisher kaum wahrgenommen worden, dass nach Einschätzung verschiedener Krankenhausärzte eine etwas längere Dauer der Influenzawelle im Herbst zu Engpässen in der intensivmedizinischen Versorgung, insbesondere bei der extrakorporalen Membranoxygenierung, geführt hätte. Die Belastungen einzelner Krankenhäuser waren, selbst innerhalb eines Berliner Klinikverbundes, sehr unterschiedlich. Der influenzabedingte Personalausfall betraf das Reinigungspersonal am stärksten und unterschied sich an den verschiedenen Standorten deutlich. 
Diese Einzelbeobachtung verdeutlicht die Notwendigkeit, die Auslastung der Versorgungsstrukturen zeitnah unter Berücksichtigung nichtmedizinischen Personals zu erfassen und Verfahren für einen entsprechenden Personalausgleich sicherzustellen.

\section{Zusammenarbeit zwischen Ärzteschaft und öffentlichem Gesundheitsdienst}

Die Zusammenarbeit des ÖGD mit den Ärztekammern, den Apothekerkammern und den Kassenärztlichen Vereinigungen, wurde von den Teilnehmenden des Workshops insgesamt positiv empfunden. Die Ärzte in Klinik und Praxis wurden zwar durch ihre Verbände informiert, es ist jedoch zu prüfen, warum die Informationen dennoch nicht alle Ärztinnen und Ärzte erreicht haben. Vielfach hat die Öffentlichkeit, aber auch die Ärzteschaft aufgrund der umfangreichen Aktivitäten der Gesundheitsämter den ÖGD bewusster wahrgenommen. Dabei wurde deutlich, dass der ÖGD und die niedergelassene Ärzteschaft eine engere Arbeitsbeziehung pflegen müssen. Regional gelang die Zusammenarbeit unterschiedlich gut und war zum Teil bereits im Rahmen verschiedener Pandemieübungen gebahnt worden.

\section{Beratungsgremien}

Das RKI hatte die Influenzakommission für den Pandemiefall (Pandemiekommission) unmittelbar einberufen. Diese aus Experten verschiedener Fachgebiete zusammengesetzte Kommission hat die Aufgabe, das RKI in der Pandemie zu beraten. Gegenüber der Öffentlichkeit wurde jedoch nicht ausreichend verdeutlicht, dass diese die jeweiligen Empfehlungen von RKI und Paul-Ehrlich-Institut (PEI) fachlich unterstützt hat.

Der Gedanke, dass ein separates externes Gremium direkt die politischen Entscheidungsträger zum Beispiel auch in ethischen Fragestellungen zur Priorisierung beraten könnte, wurde diskutiert. Gleichzeitig wurde aber auch vor der Einrichtung weiterer Gremien gewarnt, da teilweise der Eindruck bestand, politische Entscheidungsverantwortung sei zu oft auf die Fachebene beziehungsweise an externe Gremien zu delegieren versucht worden. Die Arbeitsgruppe Koordination betonte das Dilemma, dass einerseits die Meinungsführerschaft größtenteils beim Bund liege, die finanzielle und exekutive Verantwortung dagegen bei den Bundesländern und Kommunen. Dies warf die Frage auf, ob koordinierende Funktionen im Falle eines Bundesländer-übergreifenden Infektionsereignisses von öffentlicher Bedeutung künftig anders geregelt werden könnten.

\section{Koordination}

Bereits frühzeitig bestand zwischen den Ländern und den Bundesbehörden Einigkeit darüber, die Infektionsschutzmaßnahmen der Lage angepasst und eher zurückhaltend einzusetzen, um das soziale und wirtschaftliche Leben nicht mehr als erforderlich zu beeinträchtigen. Dennoch wurden einige gemeinsam beschlossene Strategien in den einzelnen Ländern oder Kommunen sehr unterschiedlich umgesetzt. So trafen zum Beispiel einige Bundesländer deutlich eingreifendere und personalaufwendigere Maßnahmen im Umgang mit Flugzeugpassagieren, als sie ursprünglich zwischen den Ländern beschlossen worden waren. Teilweise waren regionale Unterschiede in den Maßnahmen aufgrund des unterschiedlichen zeitlich-geografischen Verlaufes fachlich nachvollziehbar, wie zum Beispiel die frühzeitige reaktive Schließung von Schulen. Gleichwohl vermittelten derartige Unterschiede in der Öffentlichkeit zum Teil den Eindruck eines unabgestimmten Vorgehens. Andererseits wurde es aber auch als Vorteil empfunden, dass der deutsche Pandemieplan und seine föderale Umsetzung ein flexibles und lokal angepasstes Vorgehen ermöglichte.

\section{Impfung}

\section{Impfstoffbestellung und -lieferung}

Positiv wurde hervorgehoben, dass Impfstoff vorhanden war und grundsätzlich jeder sich impfen lassen konnte. Der Impfstoff erwies sich zudem als sicher, verträglich und in etwa so rasch verfügbar, wie in den Szenarien der Pandemieplanung angenommen. Die Verwendung eines adjuvantierten Impfstoffes, eine rasch erfolgte EU-Zulassung und die Auslieferung in 1oer-Dosen führten zu einer Verkürzung der Zeit bis zur Impfstoffauslie- ferung gegenüber üblichen saisonalen Influenzaimpfstoffen und zu einer Erhöhung der wöchentlich verfügbaren Liefermenge. Im Umsetzungsprozess verzögerte sich die Auslieferung allerdings unter anderem aus produktionstechnischen Gründen, sodass in einer Phase wachsender Impfbereitschaft anfänglich nicht alle Impfstellen mit Impfstoff versorgt werden konnten. Als der Impfstoff dann schließlich in Breite verfügbar wurde, war die Krankheitslast bereits deutlich gesunken und somit ebenfalls das Impfbedürfnis der Bevölkerung.

Die Entscheidung zum Umfang der Impfstoffbestellung musste $\mathrm{zu}$ einem Zeitpunkt getroffen werden, als weder die Impfempfehlungen beschlossen, noch die Anzahl der pro Person erforderlichen Impfdosen endgültig festgelegt, noch die Impfbereitschaft der Bevölkerung verlässlich vorhergesehen werden konnten. Das finanzielle Risiko bezüglich der zu bestellenden Impfstoffmenge konnte entgegen den Erwartungen politischer Entscheidungsträger letztlich weder durch die internationalen Empfehlungen der WHOGremien noch durch die wissenschaftlichen Einschätzungen der Bundesbehörden oder durch die Befragungen der Bevölkerung verlässlich eingegrenzt werden. Zudem bestand eine außergewöhnliche internationale Situation, in der weltweit ausschließlich Staaten Impfstoff bestellten und dieser nicht auf den „freien Markt" gelangen konnte. Dadurch konnte nicht auf die üblichen Vermarktungsund Vertriebswege zurückgegriffen werden, sodass zahlreiche Sonderregelungen getroffen werden mussten.

Vor diesem Hintergrund wurde die Rolle der Impfstoffhersteller kontrovers diskutiert. Während einerseits die Enttäuschung unter anderem über die Verhandlungen zur Sicherung der Bestelloptionen und über die anfänglich nicht erfüllten Liefermengen zum Ausdruck kam, wurde auch anerkannt, dass sich der Impfstoffhersteller bezüglich der nachträglichen Reduzierung der Liefermenge verhandlungsbereit zeigte. Letztlich bestand weitgehend Einigkeit, dass - solange eine staatliche Impfstoffproduktion nicht in Betracht gezogen wird - kommerzielle Impfstoffhersteller als wesentliche Partner bei der Vorbereitung und Reaktion auf ei- 
ne Pandemie berücksichtigt werden sollten. Es ist nachvollziehbar, dass Hersteller dabei die wirtschaftlichen Risiken bezüglich Entwicklung und Produktion von Impfstoffen ebenso zu reduzieren versuchen, wie dies die Regierungen bezüglich der Bestellung tun. Es wäre aber aufgrund der jüngsten Erfahrungen auf jeden Fall sinnvoll, für die Zukunft flexiblere Verträge zu verhandeln.

\section{Impfempfehlung}

Die Impfempfehlungen wurden aufgrund der klinischen und epidemiologischen Eigenschaften der pandemischen Influenza (H1N1) 2009 im Rahmen einer risikoorientierten Empfehlung der Ständigen Impfkommission (STIKO) festgelegt und nicht - wie ursprünglich im Pandemieplan vorgesehen - als staatlich vorzunehmende Priorisierung nach Altersjahrgängen. Wenngleich es der STIKO gelang, ausreichend lange vor Verfügbarkeit des Impfstoffes eine ausführlich begründete Impfempfehlung zu veröffentlichen, wurde doch deutlich, dass die Geschäftsordnung der STIKO für eine derart komplexe und zugleich kurzfristige Impfempfehlung nicht geschaffen ist. Es lag nicht in der $\mathrm{Zu}$ ständigkeit der STIKO, Empfehlungen zur Dosierung zu geben oder Zielgruppen zur Aufrechterhaltung der öffentlichen Sicherheit und Ordnung zu priorisieren. Diese Aspekte wurden separat von RKI und PEI beziehungsweise den Landesbehörden ergänzt. Somit stellt sich die Frage, ob die Zuständigkeiten der STIKO und der Pandemiekommission besser an diese Anforderungen angepasst werden sollten.

Unabhängig davon wurde angemahnt, dass auf Grundlage der im Jahr 2009 gewonnenen Erkenntnis frühzeitig eine für die Saison 2010/2011 angepasste Impfempfehlung zur Influenza erfolgen sollte.

\section{Kostenerstattung}

Es wurde angemerkt, dass die Rechtsverordnung, in der die Krankenkassen zur Übernahme der Impfstoffkosten verpflichtet wurden, erst spät erlassen wurde. Diese Verzögerung hat wiederum die Verhandlungen zwischen Ländern, Kostenträgern und Ärzten zum Abschluss einer Impfvereinbarung beeinträchtigt. Zudem wird im Nachhinein deutlich, dass diese Verordnung Details zu regeln versucht hat, die letztlich unnötig und sogar kontraproduktiv waren. Die in der Verordnung bereits detailliert aufgeführten Indikationsgruppen hatten gegenüber der später erstellten STIKO-Empfehlung geringfügige Unterschiede, was zu Verwirrung bei der Ärzteschaft und in der Öffentlichkeit beigetragen hat. Auch hat es sich als ungünstig erwiesen, dass ein Richtwert für die Kostenerstattung in der Verordnung angegeben wurde, weil das die Verhandlungen einer bedarfsgerechten Regelung verzögert hat. Der Sachverhalt wurde zusätzlich verkompliziert, indem die Verhandlungen über die Kostenerstattungen nicht bundeseinheitlich, sondern jeweils zwischen den einzelnen Bundesländern und den jeweiligen regionalen Kassenärztlichen Vereinigungen geführt wurden. Dies führte zu unterschiedlichen Abrechungsregelungen innerhalb Deutschlands und somit zu Unmut in Teilen der Ärzteschaft.

Die Bundesländer verfolgten bezüglich der Impflogistik unterschiedliche Konzepte und wichen aufgrund der epidemiologischen Situation und veränderter planerischer Grundlagen von den jeweiligen Pandemieplänen ab. Am Beispiel Schleswig-Holsteins wurde auf dem Workshop beschrieben, welche vielschichtigen Verhandlungen mit impfenden Ärzten, Apotheken und Logistikunternehmen kurzfristig erforderlich waren. Dies hat Ressourcen gebunden, Zeit gekostet und eine frühzeitige und transparente Darstellung der Impfstrategie erheblich erschwert. Als die Logistik schließlich organisiert war, blieb die Menge des lieferbaren Impfstoffs zunächst deutlich hinter den ursprünglichen Ankündigungen des Herstellers und der Kapazität des Verteilungssystems zurück.

Unstrittig ist, dass Eindosisampullen besser zu handhaben sind als die im Herbst 2009 verwendeten 1oer-Ampullen, insbesondere wenn nicht in zentralen Impfstellen, sondern über niedergelassene Arztpraxen geimpft wird. Zudem würden damit die Notwendigkeit eines Thiomersal-Zusatzes und die Diskussion um seine Verträglichkeit überflüssig. Vor allem die Schwierigkeit in niedergelassenen Praxen, nicht immer acht bis zehn Impflinge für die Verwendung einer 1oer-Ampulle organisieren zu können, hat vielfach dazu geführt, dass impfwillige Patienten spät oder überhaupt nicht geimpft wurden.

\section{Haftungsregelungen}

Durch die behördliche Beauftragung der niedergelassenen Ärzte im Rahmen der Impfkampagne - ob in der allgemeinärztlichen Praxis oder einer zentralen Impfstelle - war sichergestellt, dass etwaige Schadensersatzansprüche im Rahmen von Impfschäden nicht gegenüber dem impfenden Arzt, sondern gegenüber der beauftragenden staatlichen Institution geltend gemacht werden müssen. Diese Tatsache und die bestehende Entschädigungsregelung durch die Landesversorgungsämter bei möglichen Impfschäden wurden positiv bewertet. Allerdings waren beide Sachverhalte in der Ärzteschaft nicht ausreichend bekannt, sodass gegensätzliche öffentliche Äußerungen diesbezüglich zur Skepsis gegenüber den Pandemieimpfstoffen beitrugen.

\section{Rolle der niedergelassenen Ärzteschaft}

Bezüglich der Impflogistik blieb die jeweilige Rolle der niedergelassenen Ärzte einerseits und des ÖGD andererseits lange unklar. Zuständigkeiten wurden regional sehr unterschiedlich und offenbar nicht überall für beide Seiten befriedigend geregelt. Vielfach sah sich der ÖGD - bedingt durch die Bindung der gesamten personellen Ressourcen im Geschehen - nicht in der Lage, Impfungen selber durchzuführen oder in zentralen Impfstellen mithilfe hinzugezogener Ärzte zu organisieren. Aufgrund der bestellten 1oer-Dosen und der Strategie der Pandemiepläne lag zunächst eine zentrale Verimpfung nahe. Anders als in der Pandemieplanung vorgesehen, erfolgte jedoch eine Indikationsimpfung aufgrund individueller Risikoabwägung, was wiederum für eine Impfung durch Hausärzte sprach. Bezüglich künftiger Pandemieplanungen haben die Erfahrungen im Herbst 2009 auch gezeigt, dass sich eine staatlich zu verantwortende Priorisierung der Impfung bestimmter Funktionsträger im Gegensatz zu einer medizinisch begründeten Indikationsstellung über die niedergelassene Ärzteschaft kaum umsetzen lässt. Sofern die Priorisierung bestimmten Berufsgruppen entspricht, lässt sich diese über entsprechende Belieferung betriebsärztlicher Einrichtungen erreichen. Für ähnliche Situationen wie im Herbst 2009 muss die 
Rolle der niedergelassenen Ärzteschaft besser in der Pandemieplanung berücksichtigt werden.

\section{Erfassen von Impfquoten und Impfstoffsicherheit}

Im Oktober 2009 hatte im Rahmen einer Umfrage in Deutschland etwa ein Drittel der befragten Erwachsenen angegeben, sich impfen lassen zu wollen. Nach aktuellen Schätzungen des RKI haben letztlich aber weniger als 15\% der chronisch Kranken und weniger als 20\% des medizinischen Personals tatsächlich eine Impfung gegen das Pandemievirus erhalten; in der Gesamtbevölkerung haben sich etwa $7,5 \%$ aller Personen impfen lassen. In Irland, Schweden und den Niederlanden wurden dagegen zwei- bis fünfmal so hohe Impfquoten in den entsprechenden Zielgruppen erreicht.

Drei Monate nach der eigentlichen Impfaktivität haben immer noch nicht alle Bundesländer Informationen darüber, welche Alters- und Indikationsgruppen zu welchem Anteil, wann und mit welchem Impfstoff geimpft wurden. Die Erfassung unerwünschter Impfwirkungen steht grundsätzlich vor dem Problem, bei der Vielzahl der Verdachtsmeldungen die Kausalität zwischen Impfung und Beschwerdebild im Einzelfall zeitnah zu prüfen, insbesondere wenn bei nicht bekannter Anzahl von Impfungen Häufigkeiten nicht ermittelt werden können. Dadurch wurde die Vermittlung der Bewertung eingegangener Impfschadensmeldungen für das PEI erschwert.

\section{Kommunikation}

\section{Kommunikation zwischen den Institutionen}

Der hohe Informationsbedarf von Öffentlichkeit und Fachöffentlichkeit erwies sich als eine Herausforderung für alle Ebenen. Dieser war nicht immer proportional zur jeweils aktuellen gesundheitlichen Bedrohung. Das RKI hat diesen Bedarf unter anderem durch umfassende, täglich aktualisierte Lageberichte bedient. Bund, Länder und Kommunen, Ärztekammern und Kassenärztliche Vereinigungen hatten ebenfalls Hotlines eingerichtet und auf ihren Internetseiten Informationen zur Verfügung gestellt. Die initiale Frühwarnfunktion auf internationaler Ebene hat gut funktioniert. Im Vergleich zur SARS-Epidemie 2003 gelang es der WHO dieses Mal jedoch nicht so gut, systematisch aufgearbeitete Informationen über klinische Verläufe und epidemiologische Charakteristika aus den zuerst betroffenen Ländern zur Verfügung $\mathrm{zu}$ stellen. Hilfreicher waren dagegen die Informationen des Europäischen Zentrums zur Prävention und Kontrolle von Krankheiten (ECDC). Der Informationsfluss innerhalb der Bundesländer als auch zwischen den Einrichtungen des Bundes und der Länder wurde von den Teilnehmern als gut empfunden. Die zahlreichen Telefonkonferenzen haben sich als effektives Instrument für die Abstimmungsprozesse erwiesen.

\section{Vermittlung von Entscheidungen und Unsicherheiten}

Eine besondere Herausforderung für die Behörden war es, von der ersten Ankündigung der pandemischen Welle im April 2009 bis zu ihrem Auftreten im Oktober 2009 die Führung in der Bereitstellung von Informationen zu behalten, insbesondere auf der Basis kaum belastbarer Daten. Diese lange Zeitspanne wurde nicht ausreichend genutzt, um frühzeitig alle für eine an diese Pandemie angepasste Impfkampagne nötigen Kräfte zu mobilisieren. Die Wissenschaft, besonders die Epidemiologie und die Vakzinologie, war eine wesentliche Grundlage bei der Beratung der Entscheidungsträger. Allerdings mussten die Einschätzungen über die Zahl der benötigten Impfstoffdosen und die Frage der in der Bevölkerung vorbestehenden Immunität im Verlauf auch korrigiert werden.

Ein weiteres Problem der Kommunikation war, dass sowohl bei Fachleuten als auch in der Öffentlichkeit der Begriff der Pandemie mit der Vorstellung einer „Großschadenslage“ verknüpft war. Stattdessen entwickelte sich selbst für Experten überraschend ein Pandemiegeschehen, das bislang gegenüber der saisonalen Influenza keine deutlich höhere Krankheitslast in der Bevölkerung erkennen lässt. Die immunologischen und virologischen Faktoren hierbei werden in der Wissenschaft noch diskutiert.

Die überarbeiteten Pandemiepläne müssen künftig nicht nur schwere, son- dern auch weniger einschneidende Pandemien besser berücksichtigen. Vor diesem Hintergrund hätte das RKI deutlicher kommunizieren müssen, dass der bisherige Verlauf der Pandemie unerwartet moderat war, statt wiederholt vor der Möglichkeit einer mit einer Mutation des Virus einhergehenden Pathogenitätszunahme zu warnen. Das Verständnis des Prozesses der Impfstoffzulassung und seiner notwendigen Besonderheiten im Vergleich zum Zulassungsverfahren saisonaler Influenzaimpfstoffe oder therapeutischer Arzneimittel war in der Fachöffentlichkeit wenig verbreitet. Hier hätte das PEI aktiver und transparenter kommunizieren sollen. Auch nicht beeinflussbare Faktoren wie die Ausbeute der Impfstoffproduktion oder die unvermeidbare Unsicherheit bezüglich der Anzahl benötigter Impfdosen sollten künftig frühzeitig erläutert werden.

Lageänderungen und die daraus folgenden Strategieanpassungen hatte das RKI mit den Landesbehörden abgesprochen und im Epidemiologischen Bulletin sowie in Infobriefen an die Gesundheitsämter angekündigt [9]. Diese Informationen waren aber nicht allgemein verständlich genug gefasst und der Öffentlichkeit sowie der Ärzteschaft nicht aktiv vermittelt worden, sodass diese sich von den Änderungen überrascht fühlte.

Auch der Bund hatte schwer vermittelbare Prozesse und Entscheidungen nicht präventiv erläutert. Eine solche Erläuterung wäre zum Beispiel bei der Entscheidung nötig gewesen, für die allgemeine Bevölkerung einen adjuvantierten SpaltImpfstoff zu verwenden und für Bundesbedienstete aber davon abweichend einen nicht adjuvantierten Ganzvirus-Impfstoff. Die Vertraulichkeit der Verträge zur Impfstoffbestellung und ihr Zustandekommen hatten zusätzlich Spekulationen und Argwohn provoziert. Auch die im Zusammenhang mit der Impfstoffbestellung oben bereits beschriebenen Sachzwänge hätten über geeignete Informationskanäle auf eine Weise vermittelt werden sollen, die von der Ärzteschaft und der Öffentlichkeit auch wahrgenommen wird.

\section{Akzeptanz der Impfung}

Bereits vor dem Auftreten der pandemischen Influenza $\left(\mathrm{H}_{1} \mathrm{~N}_{1}\right) 2009$ war eine kritische Haltung zu Impfungen in Teilen der 
Ärzteschaft und den Medien deutlich geworden, etwa nach intensiven Diskussionen um die Impfung gegen das humane $\mathrm{Pa}$ pillomavirus (HPV) sowie um mögliche Interessenkonflikte einzelner STIKO-Mitglieder. Insbesondere bei der Influenzaimpfung ergibt sich jedoch die Indikation zur Impfung nicht nur aus der individuellen Nutzen-Risiko-Abwägung des Impflings, sondern unter anderem auch aus der Verantwortung gegenüber vulnerablen $\mathrm{Pa}$ tienten oder Familienmitgliedern. Dies wird offenbar selbst in der Ärzteschaft nicht hinreichend akzeptiert. Wenn sich selbst ärztliches und pflegendes Personal zu großen Teilen nicht impfen lässt, erscheint eine mangelnde Impfbereitschaft bei Patienten weniger verwunderlich. Selbst bei politischen Meinungsbildnern war zudem nicht überall der Gedanke etabliert, dass eine Impfstoffbestellung für den Pandemiefall im Kern eine vorsorgliche Maßnahme ist: Sie wird - wie eine Unfallversicherung oder eine Feuerwache - zwar nur im seltenen Ernstfall dringend gebraucht, muss aber in jedem Fall vorgehalten und bezahlt werden. Der lange Entscheidungsprozess um den Impfstoffkauf erschwerte die Akzeptanz der Impfung als dem wichtigsten Werkzeug der Pandemievorbereitung in der Öffentlichkeit erheblich.

\section{Internet und andere Medien}

Eine zwiespältige Rolle bei der Kommunikation spielte das Internet. Einerseits hat das sehr umfangreiche Informationsangebot der Behörden im Internet sicher viele Menschen mit verlässlichen Auskünften versorgt (allein im RKI kam es zu 35 Millionen Seitenaufrufen im November 2009 gegenüber 5 Millionen im gleichen Zeitraum der Vorjahre). Andererseits ermöglichte das Internet auch die schnelle und weite Verbreitung von Serienmails mit Falschmeldungen. Zu großer Verunsicherung trug die nachweislich falsche Behauptung bei, das im Pandemie-Impfstoff enthalte Squalen sei für das sogenannte „Golfkriegssyndrom “ verantwortlich. In Zeiten rascher Meinungsverbreitung durch moderne elektronische Kommunikationsforen sollten diese Informationskanäle zur Früherkennung von Fehlinformationen und zur Verbreitung wichtiger verlässlicher Informationen stärker genutzt werden. Während der Zeit des größten Informationsbedarfs soll- ten Originalaufzeichnungen beziehungsweise Mitschriften von Pressebriefings zeitnah im Internet bereitgestellt werden, um eine effizientere und transparentere Medienarbeit zu fördern. Das Internet ist allerdings nicht für alle Personengruppen die geeignete Informationsquelle.

Sowohl im Internet als auch durch Broschüren und Plakate wurden im gesamten Verlauf der Pandemie persönlichen Hygienemaßnahmen als Möglichkeit des Infektionsschutzes betont, aufbauend auf der Aktion „Wir gegen Viren“, die bereits im März 2009 gestartet worden war (http://www.wir-gegen-viren. de). Befragungen zeigten, dass die Kenntnisse der Bevölkerung zu den Hygieneempfehlungen zur Prävention der Influenza im zeitlichen Zusammenhang mit dieser Maßnahme und dem Pandemiegeschehen messbar gestiegen sind.

Auch zum Thema Impfen wurden entsprechende Informationsmaterialien entwickelt, diese erschienen aber offenbar einerseits zu spät und wurden zum anderen nicht so massiv verbreitet wie etwa in Schweden. Filmspots wurden lediglich als Pro-bono-Schaltungen zu eher uneffektiven Sendezeiten gesendet, und Anzeigenschaltungen fanden erst zu einem Zeitpunkt statt, als offenkundig wurde, dass die Impfbeteiligung unzureichend ist. Es wurde diskutiert, ob die Akzeptanz von Patienteninformationen erhöht werden könnte, wenn diesevon anderenEinrichtungen erstellt würde als von den für die Entscheidung von Maßnahmen verantwortlichen Behörden.

\section{Rolle der Berufs- und Fachverbände}

Wissenschaftlichen und ärztlichen Fachgesellschaften kommt eine wichtige Rolle bei der kritischen Prüfung und Vermittlung von behördlichen Entscheidungen zu. Problematisch war allerdings, dass innerhalb der ärztlichen Fach- und Berufsverbände - entweder aus einfacher Unkenntnis oder aus verbandspolitischen oder persönlichen Interessen heraus - widersprüchliche Informationen und Forderungen kommuniziert wurden. Das führte in der Ärzteschaft, aber auch in der Öffentlichkeit zu Verunsicherung.

Der vom Bundesministerium für Gesundheit im Dezember einberufene Impfgipfel unter Beteiligung der relevanten ärzt- lichen Verbände war daher sehr sinnvoll, hätte jedoch bereits im Vorfeld der Impfungen stattfinden müssen, um seine volle Wirkung entfalten zu können. Im Rahmen einer repräsentativen Umfrage des RKI stimmten etwa ein Drittel der Befragten der Aussage zu oder eher zu, dass die Diskussion um die Impfung gegen Schweinegrippe die eigene Einstellung zu Impfungen im Allgemeinen negativ beeinflusst habe. Es steht daher zu befürchten, dass das Misstrauen der Bevölkerung durch die Informationsmaßnahmen im Herbst vonseiten der Bundesbehörden nicht ausreichend abgebaut werden konnte.

Bundes- und Landesbehörden hatten die ärztlichen Fachverbände über ihre Funktion als Berater in der Pandemiekommission hinaus nicht ausreichend als Multiplikatoren in die Aufklärungsarbeit eingebunden. Die meisten Fachgesellschaften selbst wiederum hatten es lange Zeit versäumt, sich zu engagieren und zumindest fachlich offenkundig nicht haltbare Äußerungen einzelner Mitglieder auch offensiv als solche zu klassifizieren. In dieser verwirrenden Informationslage wurden von manchen impfenden Ärzten die Empfehlungen der STIKO als „Leuchtturm“ empfunden.

\section{Zukünftiges Kommunikationskonzept}

Es bestand Einigkeit, dass bei der Überarbeitung der Pandemiepläne die Kommunikation einen deutlich höheren Stellenwert erhalten muss. Über die reine Pressearbeit und Krisenkommunikation hinaus muss ein größerer Schwerpunkt auf Aufklärungsmaßnahmen gelegt werden. Aufklärungsmaßnahmen müssen auch unabhängig vom pandemischen Geschehen langfristig angelegt werden. Gleichermaßen muss auch eine vorausschauende und reaktionsfähige Kommunikationskapazität aufgebaut werden, die moderne Kommunikationskanäle und etablierte Multiplikatorennetzwerke professioneller nutzt und dabei aktuelle Erkenntnisse der Krisenkommunikation mit unterschiedlichen Bevölkerungsgruppen effektiv verarbeitet. Ein schlüssiges Kommunikationskonzept erfordert Investitionen. Es hat jedoch im Ereignisfall - und zwar nicht nur im Fall einer Influenzapandemie - das Potenzial, Ressourcen zu spa- 
ren und Vertrauen in behördliche Maßnahmen zu sichern. Zudem sollte überlegt werden, in die relevanten Entscheidungsgremien auch Kommunikationsexperten und Journalisten mit einzubeziehen, um $\mathrm{zu}$ erwartende Medienreaktionen vorab einschätzen und Vorschläge für eine effektivere Kommunikation der zu treffenden Entscheidungen frühzeitig berücksichtigen zu können.

Als kurzfristiges Ziel gilt es jetzt, sich rasch über die Kommunikationsstrategie bezüglich der im Herbst 2010 verfügbaren trivalenten Influenzaimpfung zu einigen und die erforderlichen Maßnahmen frühzeitig zu beginnen. Mittelfristig sollten darüber hinaus Maßnahmen getroffen werden, um den Kenntnisstand der Ärzteschaft in Bezug auf Impfungen allgemein zu verbessern.

\section{Ansätze für künftige Verbesserungen}

Für den weiteren Verlauf dieser und das mögliche Auftreten künftiger Pandemien, aber auch für andere akute Krankheitsausbrüche von allgemeiner gesellschaftlicher Bedeutung lassen sich aus der beschriebenen Diskussion folgende Anregungen und Verbesserungsansätze ableiten. Diese erscheinen bezüglich der Konzeption, der Strukturen, der Koordination und der Kommunikation für die künftige Pandemieplanung relevant und sollten bei künftigen Auswertungen durch die jeweils zuständigen Institutionen auf lokaler, Landes- und Bundesebene sowie im internationalen Kontext aufgegriffen werden:

\section{Konzeption}

- Die Pandemieplanung sollte deutlicher die lageabhängige statt die phasenabhängige Maßnahmenanpassung berücksichtigen.

- Die Vor- und Nachteile von Impfstofflieferung in Mehrfachdosen sollten sorgsam abgewogen und mögliche weitere Steigerungsmöglichkeiten der Lieferkapazitäten geprüft werden.

- Die Umsetzbarkeit einzelner Infektionsschutzmaßnahmen sollte - insbesondere bezüglich des Arbeitsschutzes in der ambulanten medizinischen Versorgung - kritisch überprüft werden.
- Es sollte geprüft werden, ob die Übermittlungsprozesse im Rahmen des IfSG nicht weiter automatisiert, beschleunigt und für ein umfangreiches Infektionsgeschehen flexibler gestaltet werden können.

- Die Funktion, das Mandat und die Kapazität der STIKO im Pandemiefall sollten überprüft werden.

- Der Anpassungsbedarf für Geschäftsordnung und Zusammensetzung der Pandemiekommission sollte geprüft werden. Zumindest aber sollte die Information über die Rolle und Arbeitsweise derselben verbessert werden.

- Es sollte geprüft werden, wie im Pandemiefall ethische Aspekte besser in die Politikberatung eingebracht werden können (z.B. Zuteilung verknappter medizinischer Resourcen).

\section{Strukturen}

- Ein System zur zeitnahen Erfassung der alters- und zielgruppenspezifischen Impfquote ist dringend erforderlich.

- Der ÖGD sollte stärker in die Lage versetzt werden, Impfkampagnen durchzuführen oder zu koordinieren.

- Systeme für eine schnelle und zuverlässige Information klinisch tätiger Ärzte sollten etabliert werden.

- Zur besseren Einschätzung der Krankheitslast sollte eine MortalitätsSurveillance eingerichtet werden.

- Ein Sentinel zur Erfassung schwerer Infektionskrankheiten sollte eingerichtet werden (zum Beispiel Krankenhaus-Sentinel).

- Ein im Pandemiefall handhabbares Monitoring zur Belastung der medizinischen Versorgungsstrukturen und des ÖGD sollte geschaffen werden.

- Kurzfristig realisierbare Studien zur Messung epidemiologischer Parameter sollten fest eingeplant und mit Unterstützung des ÖGD und der Ärzteschaft umgesetzt werden. Die Ergebnisse sollten zeitnah verfügbar gemacht werden.

- Ein handlungsfähiger und auch in Krisensituationen belastbarer ÖGD braucht ausreichende Ressourcen auf allen Ebenen.

\section{Koordination}

- Rechtsverordnungen zum Impfen und andere Regelungen zur Kostenerstattung sollten so frühzeitig wie möglich abgeschlossen oder zumindest für die verschiedenen Szenarien vorbereitet sein.

- Fachgesellschaften und Berufsverbände sollten auch in der aktuellen Lage früher in die Entwicklung und Vermittlung öffentlicher Empfehlungen eingebunden werden.

- Gemeinsam beschlossene Strategien sollten zwischen den Bundesländern auch einheitlich umgesetzt werden.

- Mechanismen zum Ausgleich lokal unterschiedlicher Personalbelastungen in den Versorgungsstrukturen sollten vorbereitet werden.

- Es sollte geprüft werden, ob koordinierende Funktionen im Falle eines Bundesländer-übergreifenden Infektionsereignisses von öffentlicher Bedeutung künftig anders geregelt werden könnten.

\section{Kommunikation}

- Die Kommunikation sollte ihrer Bedeutung entsprechend einen höheren Stellenwert im Pandemieplan erhalten, langfristig angelegt und angemessen finanziert werden.

- Kurzfristig sollte die Kommunikationsstrategie bezüglich der im Herbst 2010 verfügbaren trivalenten Influenzaimpfung fertiggestellt und frühzeitig aktiviert werden.

- ÖGD und die niedergelassene Ärzteschaft sollten regional und gemeinsam die Erfahrungen insbesondere in den Bereichen Koordination und Kommunikation aufarbeiten.

- Der Ärzteschaft und dem ÖGD sollte besser vermittelt werden, dass Surveillance-Maßnahmen auch zur Erstellung und Validierung von Präventionsempfehlungen erforderlich sind.

- Medizinisches und Pflegepersonal sollten über die reine Informationsübermittlung hinaus in einem sachlichen Meinungsbildungsprozess vom Sinn öffentlicher Impfempfehlungen überzeugt werden. 
- Entscheidungsprozesse und Sachzwänge der Behörden sollten vorausschauender und transparenter kommuniziert werden.

- Berufs- und Fachverbände sollten nicht nur bezüglich behördlicher Empfehlungen ihre Kompetenz kritisch einbringen, sondern auch bezüglich der öffentlichen Stellungnahmen ihrer Sprecher wissenschaftliche Maßstäbe sicherstellen.

- Strategieanpassungen (sowohl Verschärfung wie auch Deeskalation von Maßnahmen) sollten den Betroffenen und der Öffentlichkeit verständlicher und vorausschauender angekündigt und erläutert werden.

- Entscheidungsprozesse zur Impfstoffbestellung und Verfahren zur Impfstoffzulassung sollten transparenter kommuniziert werden.

- Bereits bestehende Regelungen bezüglich Schadensersatzansprüchen und Entschädigungsleistungen im Falle von Impfschäden sollten besser kommuniziert werden.

- Moderne Kommunikationsforen sollten zur Früherkennung von Fehlinformationen und zur Verbreitung wichtiger Informationen stärker genutzt werden.

- Originalaufzeichnungen beziehungsweise Mitschriften von Pressegesprächen sollten zeitnah im Internet bereitgestellt werden.

- Entwickelte Informationsmaterialien wie Broschüren, Plakate, Anzeigen und Filmspots sollten frühzeitiger und breiter eingesetzt werden.

\section{Fazit}

Der Workshop fand in offener Atmosphäre statt, in der es zu einem lebhaften Austausch von Sichtweisen und Erfahrungen kam. Dabei wurden konkrete Verbesserungsvorschläge für die Pandemieplanung geäußert, die in den zuständigen Einrichtungen und Gremien aufgegriffen, bewertet und vertieft werden müssen. Diese Pandemie hat die Wissenschaft und die Behörden auf allen Ebenen vor große Herausforderungen gestellt, obwohl sie im Vergleich zu früheren Pandemien bislang nicht sehr schwerwiegend verlief. Die Fachkräfte in der medizinischen Ver- sorgung, dem ÖGD und den Bundesbehörden waren durch ihr Engagement in der Lage, gewisse planerische, strukturelle und kommunikative Herausforderungen und Defizite zu kompensieren. Aber es wurde auch deutlich, dass diese Kompensationsmechanismen bei einer anders gearteten epidemiologischen Lage leicht an die Grenzen der Kapazität geraten können. Es hat sich gezeigt, dass ein wirksamer und verträglicher PandemieImpfstoff wenige Monate nach dem Auftreten eines neuen Influenzavirus verfügbar sein kann, wenngleich zu Beginn mit Knappheit zu rechnen ist. Auch die Organisation der Verteilung des Impfstoffs und die Einbeziehung der Ärzteschaft müssen unter Nutzung bestehender Strukturen vereinfacht werden. Das Geflecht von Zuständigkeiten ist in Deutschland derart komplex, dass die Verhandlungs- und Entscheidungsprozesse in Bezug auf Impflogistik und Impfkostenerstattung auch für Situationen wie im Herbst 2009 vorbereitet sein müssen. Vorab etablierte Erfassungssysteme im Sinne von Surveillance, Monitoring und geplanten Ad-hoc-Studien können helfen, wichtige Fragen für die Politikberatung frühzeitiger und Ressourcen sparender zu klären. Allgemein müssen Kenntnis und Verständnis der Ärzteschaft und der Öffentlichkeit bezüglich der Ziele, des Nutzens und der Risiken von Impfungen und Infektionsschutzmaßnahmen gefördert werden. Nicht zuletzt wurde offenkundig, dass die Kommunikation über getroffene und geplante Entscheidungen und Maßnahmen transparenter und vorausschauender betrieben werden muss. Nur mit Investitionen in eine Verbesserung der oben genannten Bereiche kann es gelingen, im Rahmen einer nächsten Pandemie das Vertrauen der Bevölkerung zu gewährleisten und ihren gesundheitlichen Schutz wirksam und kosteneffektiv zu sichern.

\section{Korrespondenzadresse}

\section{PD Dr. G. Krause}

Abteilung für Infektionsepidemiologie,

Robert Koch-Institut

Postfach 650261

13302 Berlin

KrauseG@rki.de
Danksagung. Wir möchten allen Teilnehmern des Erfahrungsaustausches für ihr Engagement und die konstruktiven Diskussionen danken. Da viele Teilnehmer stellvertretend für viele weitere Kollegen anwesend waren, möchten wir den Dank auf all diejenigen ausdehnen, die dazu beigetragen haben, dass die gesammelten Erfahrungen miteinander geteilt werden konnten.

Für die Vorbereitung des Workshops geht besonderer Dank an Jessica Bielecke. Wertvolle Hilfe bei der Durchführung leisteten die RKI-Mitarbeiter Udo Buchholz, Silke Buda, Tim Eckmanns, Walter Haas, Dorothea Matysiak-Klose, Romi Pohland, Sabine Reiter, Annicka Reuss, Franziska Schwarz, Dietmar Walter. Wir danken ebenfalls dem Bundesministerium für Gesundheit für die Unterstützung der Initiative.

Interessenkonflikt. Der korrespondierende Autor weist auf folgende Beziehungen hin: Über ihre jeweiligen Funktionen als Mitarbeiter einer Institution oder in Vertretung eines Verbandes hinaus haben die Autoren folgende Interessenkonflikte angegeben: B. Gärtner, C. Träder: Vortragshonorare von GlaxoSmithKline; J. Becker, V. Stollorz: Honorar des RKI im Rahmen des Workshops; O. Razum: Honorar des RKI im Rahmen dieses und anderer Workshops, Werkverträge mit dem RKI und dem AOK-Bundesverband zu Themen der Gesundheitsberichterstattung, Werkverträge und Vortragshonorare des Bundesministeriums für Arbeit und Soziales.

\section{Literatur}

1. $C D C$ (2009) Swine influenza $A(H 1 N 1)$ infection in two children - Southern California, March-April 2009. MMWR Morb Mortal Wkly Rep 58(15):400-402

2. WHO (2009) Declaration of WHO Phase 5. Statement by WHO Director-General, Dr Margaret Chan, 29 April 2009. http://www.who.int/mediacentre/news/statements/2009/h1n1_20090429/en/

3. RKI (2009) Möglicher Verlauf einer Epidemie durch das Neue Influenzavirus A/H1N1 in Deutschland und Auswirkungen präventiver Maßnahmen des Öffentlichen Gesundheitsdienstes. Epid Bull 22:219-223

4. Gilsdorf A, Poggensee G (2009) Influenza A(H1N1)v in Germany: the first 10,000 cases. Euro Surveill 14(34)

5. Arbeitsgemeinschaft Influenza (AGI) (2010) Influenza-Wochenbericht. http://www.influenza rki de/index html?c=bericht

6. Schrenk B von, Grambs W (2008) LÜKEX 2007: Wichtige Erkenntnisse für strategisches Krisenmanagement und nationale Pandemieplanung. Praxis im Bevölkerungsschutz 1:157-166

7. Nationaler Pandemieplan - Stand Mai 2007. http:// www.rki.de $>$ Infektionskrankheiten $\mathrm{A}-\mathrm{Z}>\mathrm{Neue}$ Influenza $\mathrm{A} / \mathrm{H} 1 \mathrm{~N} 1$

8. Verordnung über die Meldepflicht bei Influenza, die durch das erstmals im April 2009 in Nordamerika aufgetretene neue Virus ( Schweine-Grippe") hervorgerufen wird (SchwIMPV) vom 30.4.2009. http://www.gesetzeim-internet.de/bundesrecht/schwimpv/gesamt.pdf

9. RKI (2009) Modifikationsmöglichkeiten der Strategie zur Bekämpfung/Eindämmung der Neuen Influenza A/H1N1 in Deutschland in Abhängigkeit von der Entwicklung der Ausbreitung und der Schwere der Erkrankungen. Epid Bull 27:259-262 